\title{
Mini Bone Block Distraction Subtalar Arthrodesis (SAMBB) in the Management of Acquired Adult Flatfoot with Subtalar Arthritis: A Modification to the Grice-Green Procedure
}

\author{
Massimiliano Mosca ${ }^{1}$ Silvio Caravelli ${ }^{1}$ Francesca Vannini ${ }^{2} \quad$ Camilla Pungetti $^{3} \quad$ Giuseppe Catanese $^{1}$ \\ Simone Massimi ${ }^{1}$ Mario Fuiano $^{1}$ Cesare Faldini $^{2}$ Sandro Giannini ${ }^{4}$ \\ ${ }^{1}$ II Clinic of Orthopaedics and Traumatology, IRCCS Istituto \\ Ortopedico Rizzoli, Bologna, Italy \\ 2I Clinic of Orthopaedics and Traumatology, IRCCS Istituto \\ Ortopedico Rizzoli, Bologna, Italy \\ ${ }^{3}$ Department of Orthopaedics and Traumatology, Ospedale Maggiore \\ "A. Pizzardi”, Bologna, Italy \\ ${ }^{4}$ Alma Mater Studiorum, Bologna University, IRCCS Istituto \\ Ortopedico Rizzoli, Bologna, Italy \\ Address for correspondence Silvio Caravelli, MD, II Clinic of \\ Orthopaedics and Traumatology, IRCCS Istituto Ortopedico Rizzoli, \\ Bologna, Italy (e-mail: doct.car@gmail.com). \\ Joints 2019;7:64-70.
}

\begin{abstract}
Adult acquired flatfoot encompasses a wide range of deformities. The goal of surgical treatment of adult acquired flatfoot is to achieve proper alignment of the hindfoot and maintain as much flexibility as possible in the foot and ankle complex; nevertheless, if subtalar arthritis is present, subtalar arthrodesis is usually mandatory. A lateral approach over the sinus tarsi is performed and subtalar joint is prepared removing remaining cartilage, reduced up to 5 degrees of heel valgus, and stabilized with a Kirschner wire. The site of insertion of the corticocancellous bone graft, harvested from the ipsilateral proximal tibia, is prepared carving a vertical groove into the talar and

Keywords

- flatfoot

- valgus deformity

- subtalar

- arthrodesis

- bone graft calcaneal edges. Additional autologous cancellous chips, harvested from the proximal tibia, can be positioned and pressed with a beater into the sinus tarsi to enhance fusion. The technique described is a modification of the extra-articular arthrodesis originally proposed by Grice. mini bone block distraction subtalar arthrodesis is a simple and effective technique which permits a subtalar fusion with a restored orientation of the hindfoot and ankle alignment in acquired flatfoot with subtalar arthritis.
\end{abstract}

\section{Introduction}

Adult acquired flatfoot encompasses a wide range of deformities. The deformity is characterized by flattening of the medial longitudinal arch, sliding in valgus of the calcaneus, abduction of the forefoot, dysfunction of the posteromedial soft tissues, including the posterior tibialis tendon, and frequently Achilles tendon retraction. ${ }^{1}$ This malalignment pattern results from a complex three-dimensional deformity of the subtalar joint, including valgus, extrarotation and dorsiflexion of the calcaneus, plantar flexion of the talus, abduction of the tarsal navicular bone, and subtalar eversion., ${ }^{2,3}$ Flatfoot spectrum may range from a subtle, easily correctable foot to a rigid, uncorrectable plano-valgus deformity. ${ }^{4}$

A wide variety of surgical procedures have been reported for the treatment of adult acquired flatfoot, considering tibialis posterior lesion stage, localization and degree of deformity, and whether the deformity is flexible or rigid. ${ }^{5}$ received

May 9, 2018

accepted after revision

October 15, 2019

published online

December 13, 2019
DOI https://doi.org/

$10.1055 / \mathrm{s}-0039-3400452$.

ISSN 2282-4324.
Copyright @ 2019 Georg Thieme Verlag

KG Stuttgart · New York
License terms

c) $(1) \$$ 


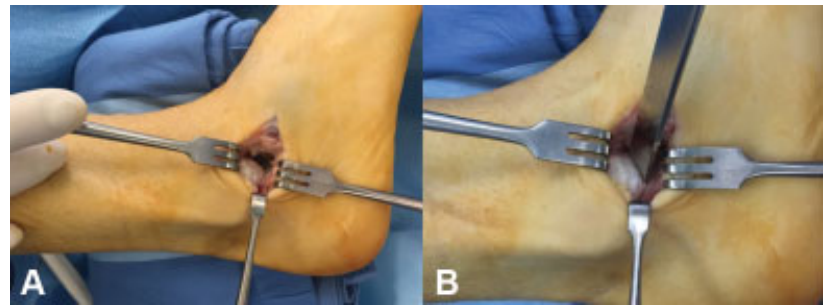

Fig. 1 (A, B) Direct visualization of the sinus tarsi and resection of the lateral process of the talus.

In cases of rigid deformity with subtalar arthritis, subtalar arthrodesis is still considered the gold standard. ${ }^{6-8}$

Subtalar arthrodesis is considered a simple and rapid, nevertheless it has to be considered mandatory to reach an adequate restoration of the hindfoot shape and the restoration of the Meary's line continuity. The residual incongruency which may result at the level of the midtarsal joint, if hindfoot shape is not adequately corrected, may lead to early arthritis progression of the ipsilateral foot joints. ${ }^{6-8}$

Grice-Green subtalar arthrodesis is a widely popular procedure. Originally performed by William Green, it was then first described in 1952 by Grice in a study on 22 patients affected by pes plano-valgus and poliomyelitis. ${ }^{9}$ Further publications of results followed. The use of metallic internal fixation was mentioned by Grice in $1959 .^{10,11}$ Since the eradication of polio, the Grice extra-articular arthrodesis has been more commonly used and the indications expanded to include spastic cerebral palsy, spina bifida, myelomeningocele, congenital vertical talus, and talocalcaneal coalition. ${ }^{3,12}$ Since the first description by Grice, several modifications have been applied to the original technique. ${ }^{13-16}$ Due to its versatility and capability to restore the shape and thickness of the hindfoot, with limited complications and no hardware required, the Grice-Green procedure may be easily used for adult flatfoot correction, when arthritis is present in the subtalar joint or the damage to the tibialis posterior requires an additional procedure of tendon augmentation with considerable invasivity, in association to the procedure of high foot realignment.

The aim of this article is to present a technical tip describing authors' modifications to the Grice-Green procedure to make it applicable to the acquired adult flatfoot.

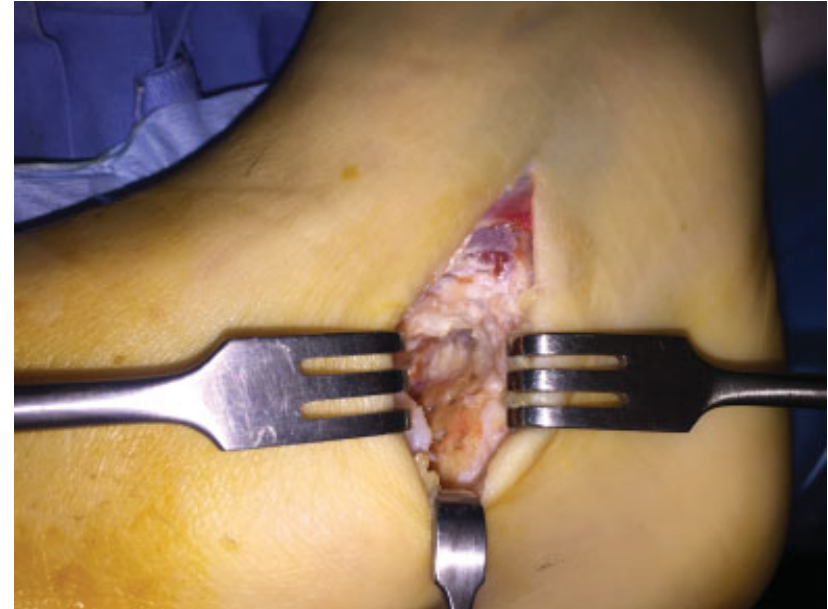

Fig. 3 Preparation of subtalar posterior facet subchondral surface by cartilage removal and drilling.

\section{Surgical Technique}

Under spinal anesthesia, the patient is placed in supine position, with feet at approximately $2 \mathrm{~cm}$ from the end of the operating table and a thigh tourniquet is applied and inflated for lower limb exsanguination, following the Association of periOperative Registered Nurses guidelines (tourniquet inflated intraoperatively to a pressure higher than the limb occlusion pressure). ${ }^{17}$

Intravenous antibiotic prophylaxis with a single dose of cephalosporin is administrated 30 minutes before exsanguination. Leg and feet affected are prepared and draped. A lateral 2.5 to $3 \mathrm{~cm}$ skin incision is performed over the sinus tarsi. The fat in the sinus tarsi is either excised or reflected and the lateral process of the talus is resected to improve exposure ( $\mathbf{- F i g . ~} \mathbf{1}$ ).

A pair of curved-bladed Mayo scissors is inserted through the sinus tarsi, directed toward the medial malleolus apex, to simulate and verify the reducibility of the deformity (-Fig. 2).

After the introduction of a laminar spreader to distract the posterior facet of the subtalar joint, the residual cartilage in debrided and the subchondral surfaces are prepared in the area under direct visual control and drilled with a Kirschner wire (K-wire) to improve the recruitment of bone marrow mesenchymal stem cells ( - Fig. $\mathbf{3}$ ).

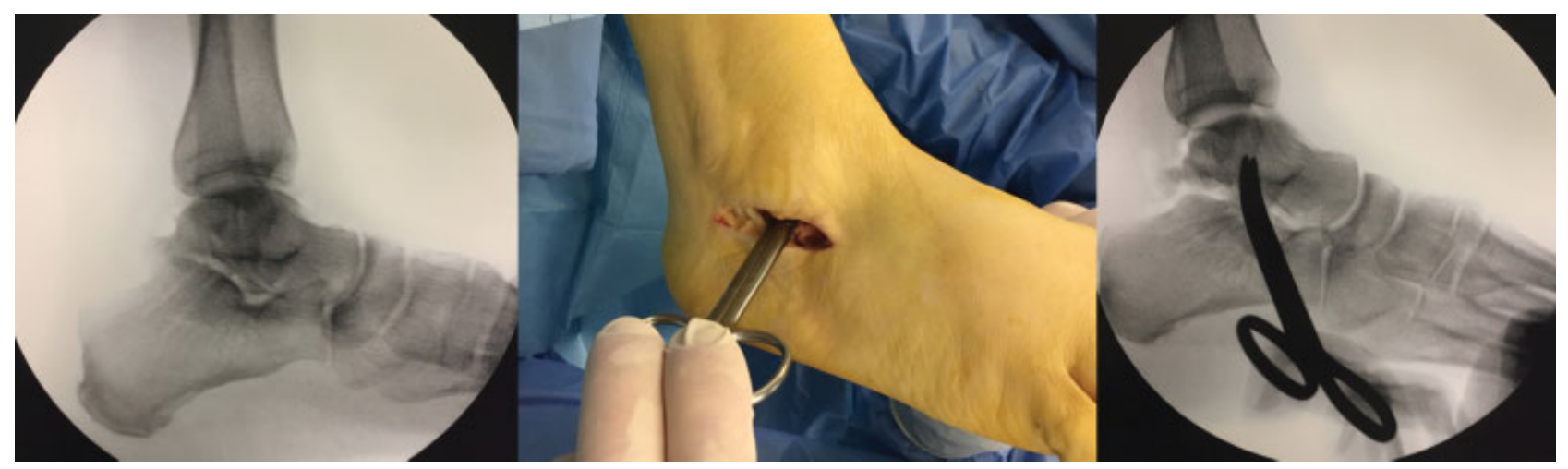

Fig. 2 Curved-bladed Mayo scissors through the sinus tarsi, verifying clinically and, if needed, radiographically the reducibility of the deformity. 


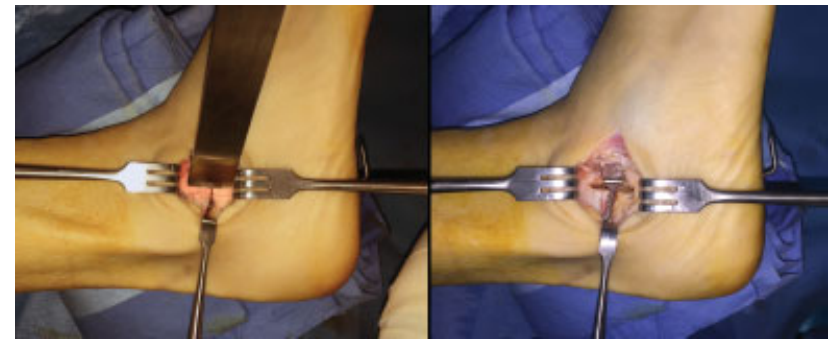

Fig. 4 With the subtalar joint manually reduced ( 5 degrees of valgus alignment, internally rotated and inverted calcaneus), the position is stabilized with a 2.5-mm Kirschner wire (K-wire).

The subtalar joint is then reduced up to 5 degrees of heel valgus. This usually requires internal rotation and inversion of the calcaneus back under the talus. The position is then stabilized with a K-wire $(2.5 \mathrm{~mm})$ positioned from distal to proximal from the calcaneus into the hindfoot ( - Fig. 4). The site where the structural graft has to be positioned is prepared with the help of an osteotome of proper size (generally a $20-\mathrm{mm}$ chisel), carving a groove into the inferior surface of the talus and into superior surface of the calcaneus where the graft has to be stably fitted in at an angle slightly posterior to vertical (-Fig. 5).

The corticocancellous graft is cut from the ipsilateral proximal tibia. ${ }^{18} \mathrm{~A}$ longitudinal incision is then centered over the anterior crest of the tibia, approximately $3.0 \mathrm{~cm}$ distal to the knee joint line (-Fig. $\mathbf{6}$ ), and dissection is carried down to the periosteum. The periosteum is incised and carefully retracted, and the cortical bone is identified on the anteromedial side of the tibia. The outline of the needed graft, usually about the same size of the osteotome used in sinus tarsi preparation, is marked on the periosteum ( $\mathbf{- F i g . ~ 7 ) . ~ T h i s ~ i s ~}$ divaricated and a standard pneumatic oscillating saw is used to cut the cortical bone. A 3.5-mm drill bit can be used to predrill the corners and lessen the risk of a corner stress riser because rounded edges are theoretically less likely to propagate. A smaller chisel is then used to complete the cuts and deliver a corticocancellous graft of proper size ( - Fig. 8). Several cubic millimeters of additional cancellous bone also can be obtained through the bone window by using a curette, harvesting it deeply in the proximal tibia (-Fig. 9). Hemostatic sponge (Spongostan; Johnson \& Johnson, New Brunswick, New Jersey, United States) is used to fill the defect. Finally, the carefully retracted periosteum is closed over the bone defect with an interrupted adsorbable no. $2-0$ suture. Subcutaneous and skin suture is then performed.

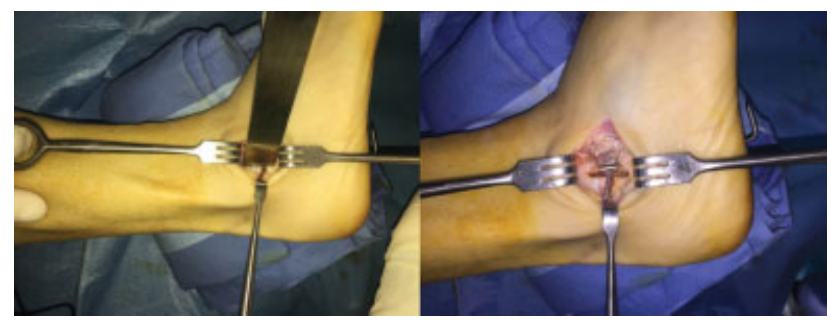

Fig. 5 Preparation of the groove on talus and calcaneus, using a 20-mm osteotome, to position the structural bone graft.

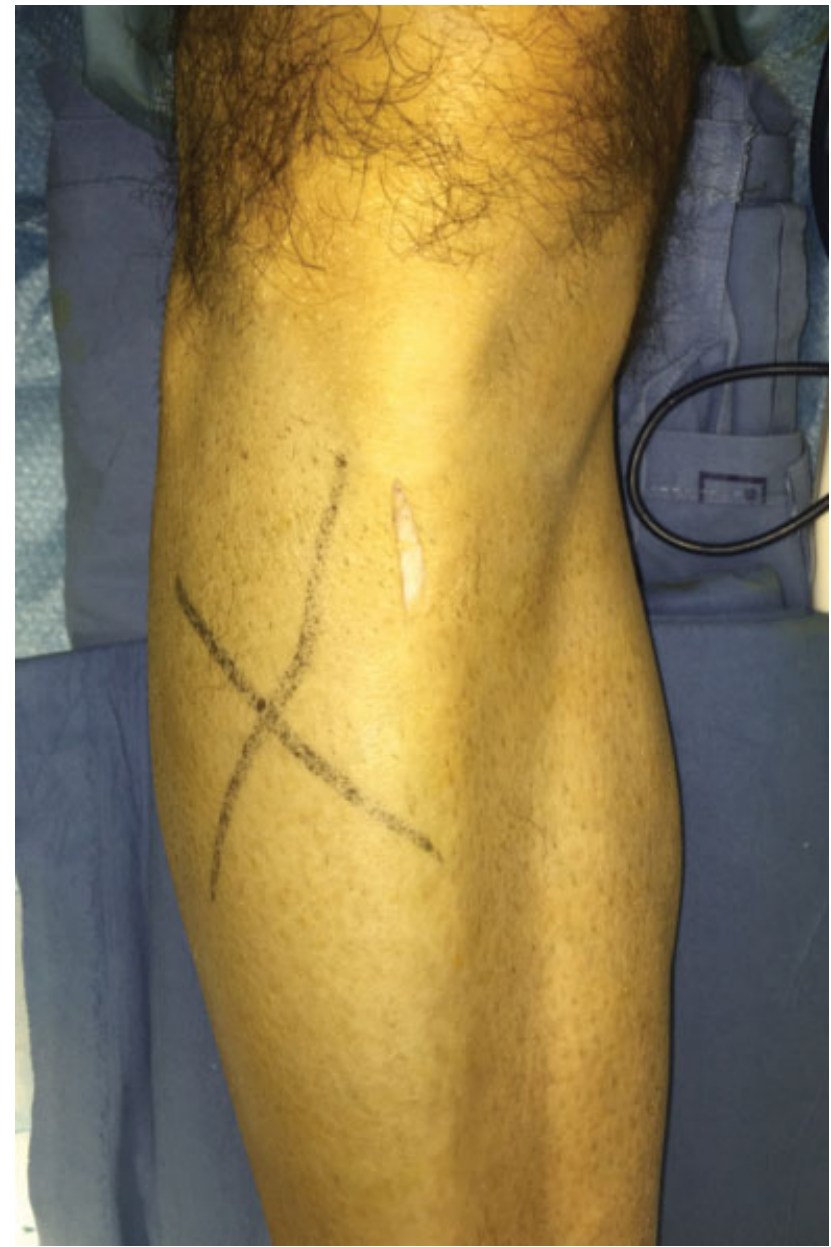

Fig. 6 Proximal tibia skin incision, $\sim 3.0 \mathrm{~cm}$ distal to the knee joint line on the anterior crest.

The corticocancellous graft harvested from the proximal tibia is prepared to appear suitable (-Fig. 10) and then positioned in a slightly vertical position into the sinus tarsi, into the previously created grooves, permitting the stability of the graft itself ( - Fig. 11). Additional autologous cancellous chips, harvested from the proximal tibia, are then positioned and pressed with a beater into the sinus tarsi to enhance fusion (-Fig. 12).

Tourniquet is deflated and tourniquet time recorded. Hemostasis is performed and skin closed, being careful of tension, with interrupted absorbable no. 3-0 suture.

\section{Additional Procedures}

Before mini bone block distraction subtalar arthrodesis (SAMBB), ankle dorsiflexion capability is evaluated maintaining the hindfoot in correction with the knee in extension. If 90 degrees cannot be obtained, an Achilles tendon lengthening by alternate subcutaneous hemisections, and subsequent forcing of the foot in dorsiflexion, is performed.

In case of hallux valgus or other forefoot deformities, a correction by S.E.R.I. (Simple, Effective, Rapid, Inexpensive) distal linear metatarsal osteotomy procedure ${ }^{19}$ and further forefoot corrections according to pathoanatomy (such as I MTP arthrodesis, bunionette correction, external toes deformities correction) are performed. 


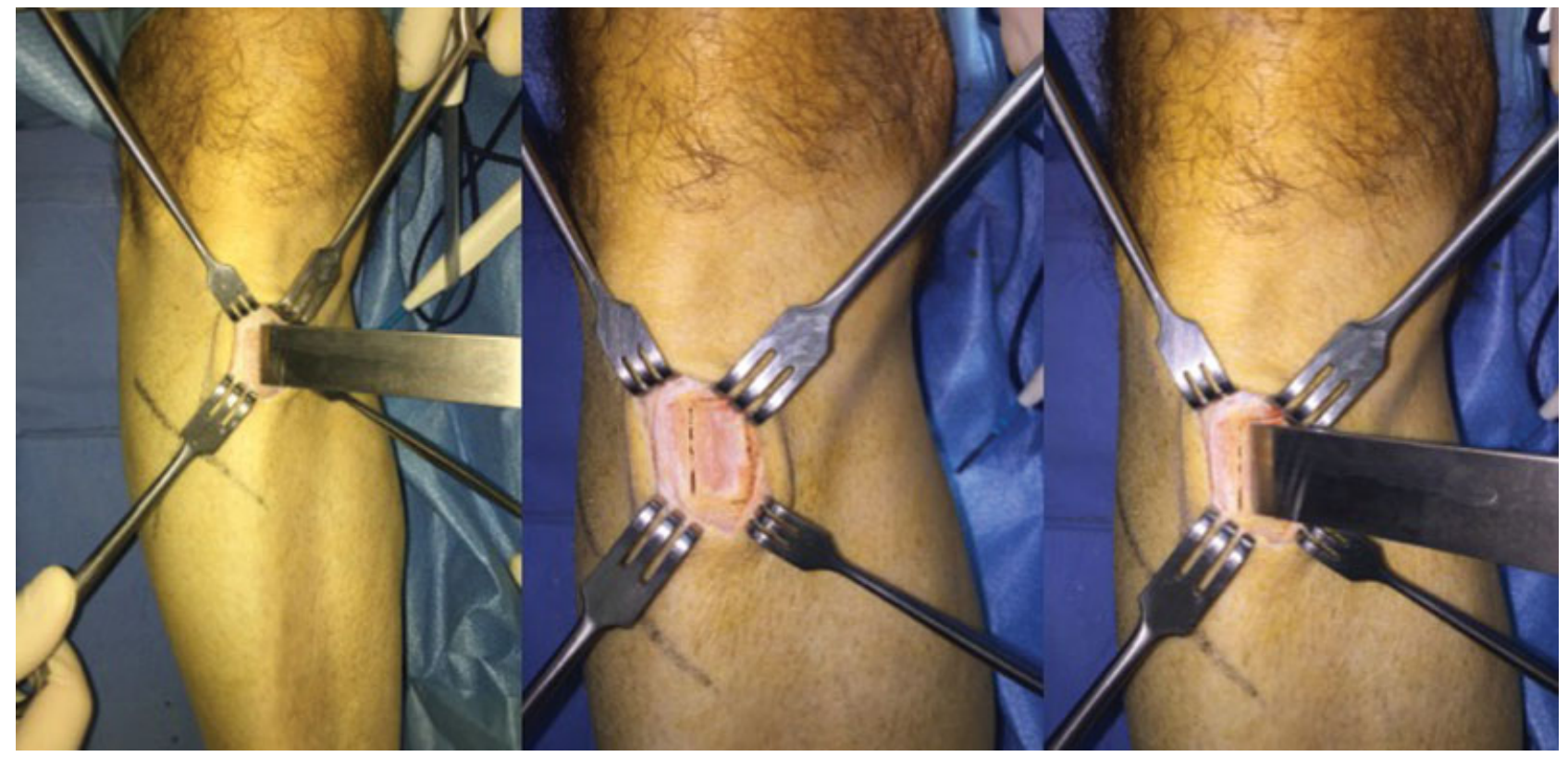

Fig. 7 Taking in consideration the dimension of the osteotome used in sinus tarsi preparation, the periosteum is incised and divaricated to be sutured after the harvest. With a traditional oscillating saw the cortical bone is cut and the corticocancellous graft is delivered.

\section{Postoperative Management}

A nonweight bearing short leg cast to protect both surgical sites is applied for 40 days, followed by a new weight bearing cast or walker boot for a further 40 days.

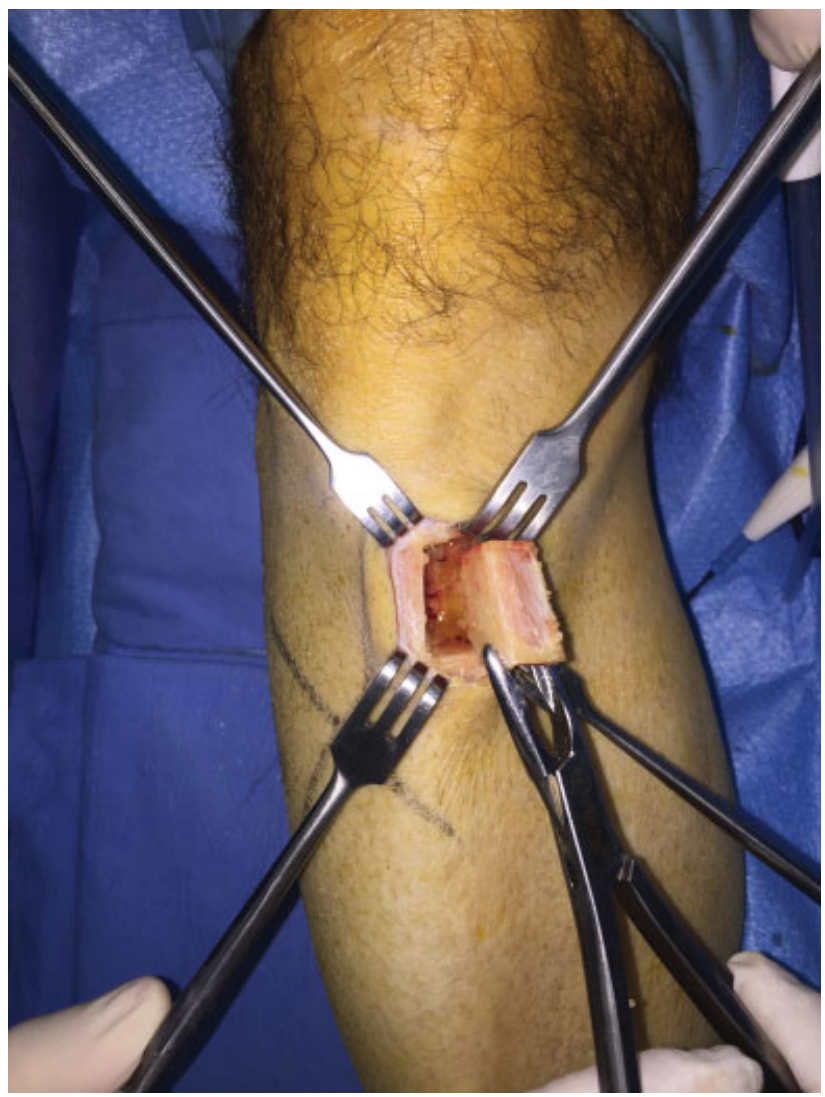

Fig. 8 The corticocancellous bone graft.

\section{Discussion}

The goal of surgical treatment of adult acquired flatfoot is to achieve proper alignment of the hindfoot and maintain as

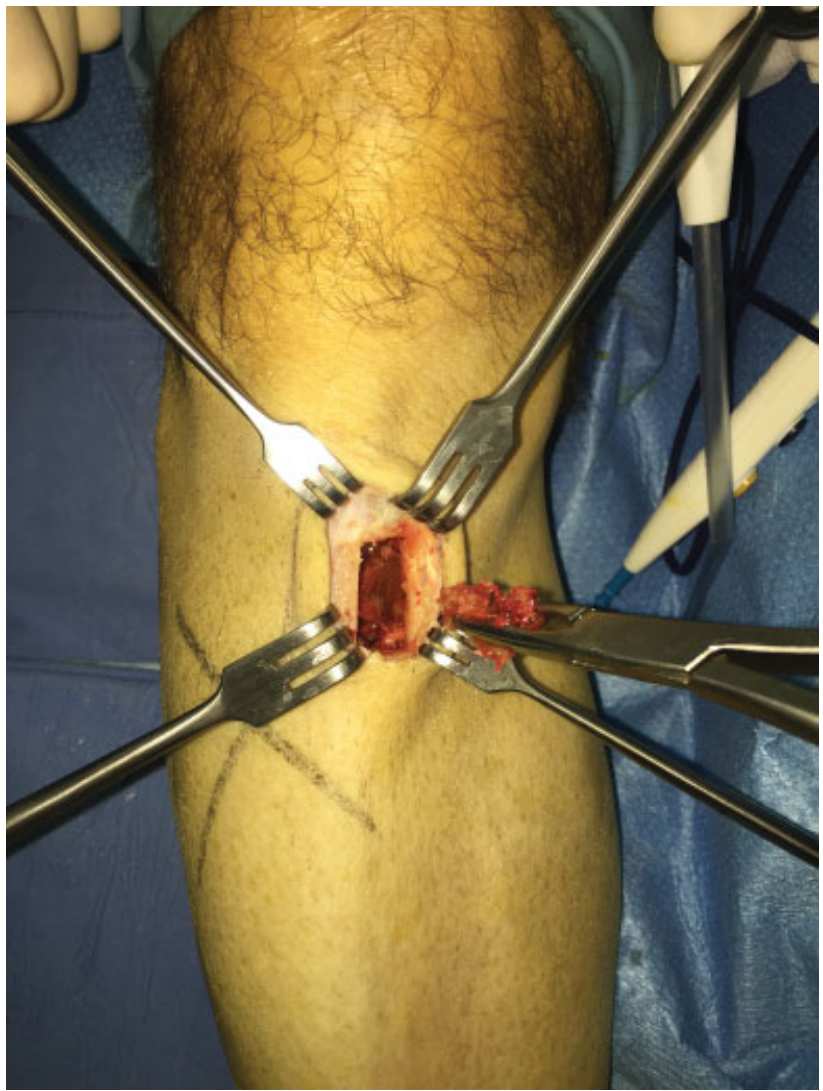

Fig. 9 Additional cubic millimeters of cancellous bone are obtained through the bone window by using a curette. 


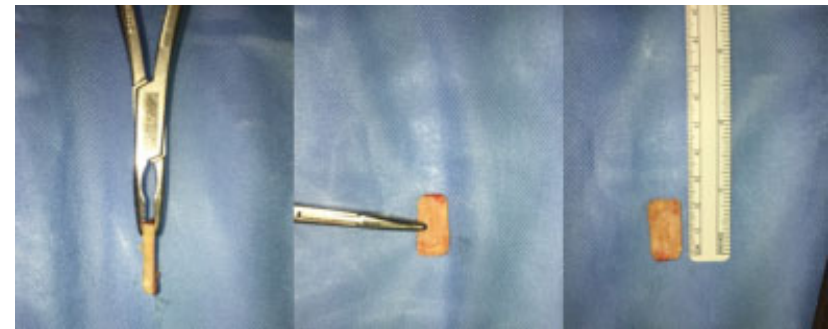

Fig. 10 The structured bone graft is finished and shaped by using a Liston bone cutting forceps or a Luer bone rongeur.

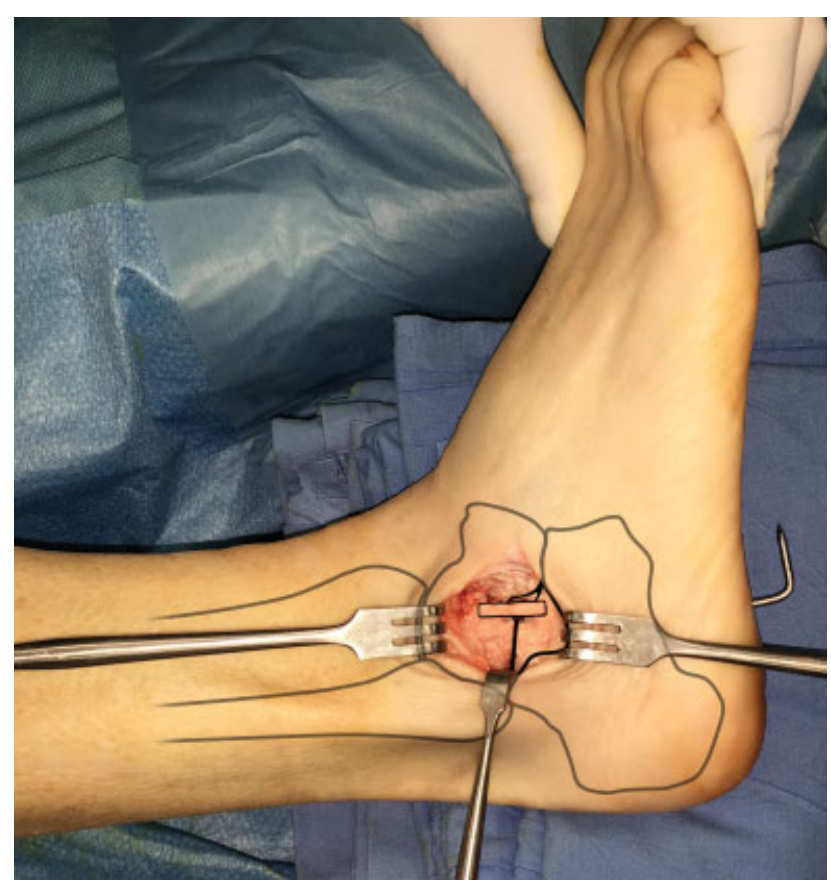

Fig. 11 The shaped bone graft is positioned into the groove by pressfit fixation. Figure shows the anatomical relations of the bone graft with the subtalar joint structures.

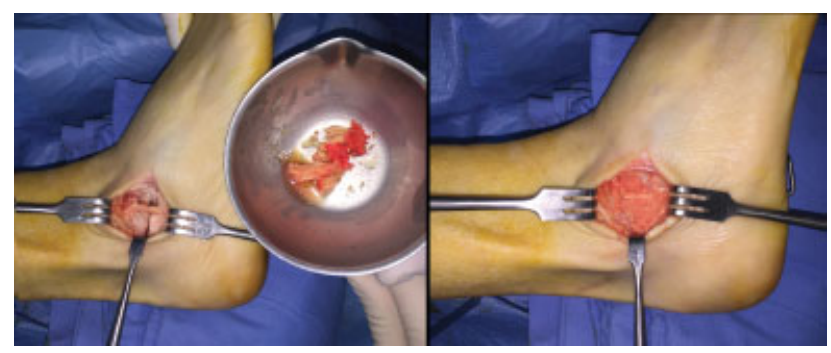

Fig. 12 Additional autologous cancellous chips, harvested from the proximal tibia, pressed with a beater into the sinus tarsi to enhance fusion.

much flexibility as possible in the foot and ankle complex; nevertheless, if subtalar arthritis is present, subtalar arthrodesis is usually mandatory.

Grice procedure has the advantage to correct flatfoot deformity restoring the correct relationship of talus with calcaneus and navicular bone; furthermore, no fixation device is required and an integration of the bone block is possible. SAMBB is indicated for adults affected by flatfoot with subtalar arthritis. Other indications are: adult flatfoot with high grade dysfunctional tibialis posterior tendon, adult flatfoot characterized by severe joint laxity, adult flatfoot with sinostosis, and adult flatfoot in neurological or syndromic clinical picture.

The key role of hindfoot alignment is widely known. Neufeld and Myerson ${ }^{20}$ reported that a subtalar arthrodesis in situ is not adequate in correcting the components of a flatfoot deformity. In fact, an arthrodesis of subtalar joint performed in a poor alignment may easily result in early arthritis of ankle and midtarsal joint, painful calcaneofibular impingement, and is in general poorly tolerated.

The capability to obtain a good hindfoot alignment is sometimes tricky. Surgical techniques regarding subtalar arthrodesis without graft insertion, require extensive cartilage removal from the articular surfaces, a quite extensive surgical approach, and the need of a fixation by screw. ${ }^{21}$

With regard to the union rate, it has been compared with fusions obtained without grafting. The use of a bone block while correcting a flatfoot by subtalar arthrodesis seems to help also in terms of rate of successful fusions obtained. In a comparative study, Chahal et $\mathrm{al}^{22}$ reported a union rate of $95 \%$ in the bone graft group compared with $65 \%$ for patients treated with an in situ subtalar arthrodesis.

The Grice-Green procedure has been widely used, with various modifications, but mostly in young patients without subtalar arthritis.

The technique described is a modification of the extraarticular arthrodesis originally proposed by Grice for the treatment of paralytic flatfeet in children. ${ }^{9}$ SAMBB is a simple and effective technique which permits a subtalar fusion with a restored orientation of the hindfoot and ankle alignment. Taking advantage of a structural bone graft fitted in as a bridge between talus and calcaneus, in a site (the sinus tarsi) that is normally empty and extra-articular, the SAMBB enables a minimally invasive and very stable arthrodesis. The addition of technical steps such as the subtalar cartilage resection under visual control and of cancellous bone chips to enhance consolidation helps the technique to be extended to other indications. Furthermore, the use of a structural bone graft harvested from the proximal ipsilateral tibia, instead of the iliac crest, considerably reduces the morbidity of this surgical procedure. ${ }^{8}$ The possibility that graft harvesting can be performed in the same surgical field and the harvesting site is included in the postoperative plaster cast is noticeable.

According to Grice, blocking the tarsal sinus open prevents the calcaneus from rotating posterolaterally and thus blocks the heel and foot from shifting into the valgus position resulting in an extremely stable arthrodesis.

The structural bone block is properly positioned in a site created with a chisel, slightly vertical; in this way, even if the graft results small and with only one cortical surface, it is very stable and permits a complete hindfoot deformity correction with no need of fixation devices (the single Kwire used for stabilization will be removed after the first cast) and very limited need of intraoperative fluoroscopy. ${ }^{23}$ As much as described for Grice procedure, union takes place very rapidly and the graft is solidly incorporated into the talus and calcaneus within 8 to 10 weeks after operation. ${ }^{10}$ 
Overcorrection with heel varus is the most common technical error reported in accounts of the Grice procedure. ${ }^{23}$ General agreement that is better to err on the side of slight valgus position of the heel at the time of operation than to overcorrect is reported. ${ }^{24} \mathrm{~A}$ manual correction of the hindfoot alignment before graft insertion, with a temporary stabilization by a K-wire and the use of the same chisel to prepare the site of graft positioning and to harvest the graft itself, may be effective technical pearls in avoiding this complication.

\section{Conclusion}

Due to aforecited characteristics, the authors consider the SAMBB viable and a safe procedure to be used in subtalar arthritis even of mild grade, in joint laxity, and in all elderly, overweight patients when a stable, not painful foot, is needed.

\section{Conflict of Interest}

None declared.

\section{Acknowledgment}

The authors would like to thank Dr. Maria Pia Cumani from Scuola di Disegno Anatomico, Rizzoli Orthopaedic Institute (Bologna, Italy) for artwork and figures.

\section{References}

1 Greisberg J, Hansen ST Jr, Sangeorzan B. Deformity and degeneration in the hindfoot and midfoot joints of the adult acquired flatfoot. Foot Ankle Int 2003;24(07):530-534

2 O'Connell PA, D'Souza L, Dudeney S, Stephens M. Foot deformities in children with cerebral palsy.J Pediatr Orthop 1998;18(06):743-747

3 Mazis GA, Sakellariou VI, Kanellopoulos AD, Papagelopoulos PJ, Lyras DN, Soucacos PN. Results of extra-articular subtalar arthrodesis in children with cerebral palsy. Foot Ankle Int 2012;33(06): 469-474

4 Meehan RE, Brage M. Adult acquired flat foot deformity: clinical and radiographic examination. Foot Ankle Clin 2003;8(03):431-452

5 Francisco R, Chiodo CP, Wilson MG. Management of the rigid adult acquired flatfoot deformity. Foot Ankle Clin 2007;12(02):317-327

6 Mann RA, Beaman DN, Horton GA. Isolated subtalar arthrodesis. Foot Ankle Int 1998;19(08):511-519
7 Johnson JE, Yu JR. Arthrodesis techniques in the management of stage II and III acquired adult flatfoot deformity. Instr Course Lect 2006;55:531-542

8 Bluman EM, Myerson MS. Stage IV posterior tibial tendon rupture. Foot Ankle Clin 2007;12(02):341-362

9 Grice DS. An extra-articular arthrodesis of the subastragalar joint for correction of paralytic flat feet in children. J Bone Joint Surg Am 1952;34 A(04):927-940

10 Grice DS. Further experience with extra-articular arthrodesis of the subtalar joint. J Bone Joint Surg Am 1955;37-A(02):246-259

11 Grice DS. The role of subtalar fusion in the treatment of valgus deformities of the feet. AAOS Instr Course Lect 1959;16:127-150

12 Høiness PR, Kirkhus E; HØness PR. Grice arthrodesis in the treatment of valgus feet in children with myelomeningocele: a 12.8-year follow-up study. J Child Orthop 2009;3(04):283-290

13 Seymour N, Evans DK. A modification of the Grice subtalar arthrodesis. J Bone Joint Surg Br 1968;50(02):372-375

14 Brown A. A simple method of fusion of the subtalar joint in children. J Bone Joint Surg Br 1968;50(02):369-371

15 Dennyson WG, Fulford GE. Subtalar arthrodesis by cancellous grafts and metallic internal fixation. J Bone Joint Surg Br 1976;58$\mathrm{B}(04)$ :507-510

16 Güven M, Tokyay A, Akman B, Encan ME, Altintaş F. Modified Grice-Green subtalar arthrodesis performed using a partial fibular graft yields satisfactory results in patients with cerebral palsy. J Pediatr Orthop B 2016;25(02):119-125

17 AORN Recommended Practices Committee. Recommended practices for the use of the pneumatic tourniquet in the perioperative practice setting. AORN J 2007;86(04):640-655

18 Vannini F, Giannini S, Vogtman J, Sparks NW, Miller SD. Technique tip: a technique for harvesting corticocancellous bone grafts from the proximal tibia. Foot Ankle Int 2006;27(08):648-649

19 Giannini S, et al. Hallux valgus surgery: the minimally invasive bunion correction (SERI). Tech Foot Ankle Surg 2003;2(01):11-20

20 Neufeld SK, Myerson MS. Complications of surgical treatments for adult flatfoot deformities. Foot Ankle Clin 2001;6(01):179-191

21 Johnson KA, Strom DE. Tibialis posterior tendon dysfunction. Clin Orthop Relat Res 1989;(239):196-206

22 Chahal J, Stephen DJ, Bulmer B, Daniels T, Kreder HJ. Factors associated with outcome after subtalar arthrodesis. J Orthop Trauma 2006;20(08):555-561

23 Ross PM, Lyne ED. The Grice procedure: indications and evaluation of long-term results. Clin Orthop Relat Res 1980;(153): 194-200

24 Bacardi BE, Rubin SZ, Turf RM. Complications of the Grice-Green operation. J Foot Surg 1989;28(04):325-332 